\title{
Genetic Test Stewardship: Making the Case for Stakeholder Collaboration Towards Efficient Testing and Billing
}

\author{
M. Edye Conway ${ }^{1}$, Cassidi Dailey Kalejta ${ }^{1}$, Darci L. Sternen ${ }^{2}$ and Ila R. Singh ${ }^{3 *}$ \\ ${ }^{\prime}$ Metis Genetics, Addison, TX, USA \\ ${ }^{2}$ Department of Laboratories, Seattle Children's Hospital, Seattle, WA, USA \\ ${ }^{3}$ Department of Pathology, Texas Children's Hospital and Baylor College of Medicine, Houston, TX, USA
}

\begin{abstract}
Laboratory stewardship programs have demonstrated an improvement in the genetic testing process. The involvement of genetics experts in the review of genetic test orders reduces inappropriate orders and their associated costs. There are many stakeholders in the genetic testing process, including patients, providers, payers, healthcare systems, and laboratories. We present four cases that illustrate common pitfalls towards optimal testing and reimbursement. These cases demonstrate current challenges in genetic testing including confusion in test selection, patient-driven orders, coverage deficiencies, and prior authorization errors. We have developed a checklist that helps navigate challenging steps in genetic testing. Additional collaboration and communication among all stakeholders are needed to improve this process.
\end{abstract}

\section{Introduction}

There are an estimated 75,000 genetic testing products on the market currently, representing approximately 10,000 unique test types. Approximately 10 new genetic tests enter the market every day [1], making genetic testing the fastest growing group of laboratory tests in recent years [2]. Many private payers have coverage criteria for a defined set of genetic tests [3-6]. However, payers frequently require prior authorization (preauthorization) which necessitates payer review of genetic tests on an individual basis, requiring the submission of the patient's clinical information and an explanation of how the requested test would alter the patient's medical management. Some hospitals have established laboratory stewardship programs [7] whereby doctoral-level consultants or genetic counselors perform a systematic review of send-out tests. Such programs address the confusion caused by a large number of genetic test options, mitigate the difficulty of test selection by non-genetic providers, and decrease the uncertainty of reimbursement. These stewardship programs have also been shown to control substantial genetic testing costs to institutions and to the healthcare system $[8,9]$. Healthcare providers, patients, payers, health systems, and laboratories are all stakeholders in the genetic testing process and outcomes. Collaboration among these stakeholders is necessary for the success of a laboratory stewardship program and for the efficient operation of the healthcare system overall.

In this paper, we present four cases that demonstrate current challenges with genetic test orders, prior authorizations, and reimbursement. We then demonstrate the benefits of having a laboratory test stewardship program. Each case has been modified for illustrative purposes, and to prevent any identification of patients.

\section{Case presentation}

\section{Case 1: Test naming}

A 50-year-old Caucasian male developed pain and redness in his right calf after a trans-Atlantic flight. His physician noted swelling and tenderness in the leg. Doppler ultrasound revealed deep vein thrombosis. The patient was treated and investigated for possible causes of venous thromboembolism (VTE). Other than the history of prolonged immobilization, the patient had no other predisposing factors for VTE. A preliminary screen for VTE, the activated protein C (APC) resistance test, was positive. His medical team wanted to know if the patient had the F5 gene Factor

*Correspondence to: Ila R. Singh, Department of Pathology, Texas Children’s Hospital and Baylor College of Medicine, Houston, TX, USA, E-mail: irsingh@ texaschildrens.org

Citation: Conway ME, Kalejta CD, Sternen DL, Singh IR (2020) Genetic Test Stewardship: Making the Case for Stakeholder Collaboration Towards Efficient Testing and Billing. J Clin Anat Pathol, 5(2): 106. DOI: https://doi.org/10.47275/2332-4864-106.

Received: August 12, 2020; Accepted: August 28, 2020; Published: September 01, 2020

Copyright: $\odot 2020$ Conway ME, et al. This is an Open Access article distributed under the terms of the Creative Commons Attribution 4.0 International License (CC-BY) (http://creativecommons.org/licenses/by/4.0/) which permits commercial use, including reproduction, adaptation, and distribution of the article provided the original author and source are credited. 
V Leiden (Leiden) variant, the most common cause of inherited VTE. The Leiden variant, c.1691G $>$ A (legacy R506Q), eliminates the APC cleavage site on Factor V, slowing down its inactivation, leading to the production of more thrombin, and thus more clots. Instead of ordering to test for the Factor V Leiden variant, the test for Factor V Level was ordered. It is possible that the wrong test was mistakenly chosen because the two tests, Factor V Leiden and Factor V Level, were adjacent to each other on the order screen. It is also possible that because the test was ordered by a trainee, they were unsure of which test to order [7]. Factor V levels are useful in cases of Factor V deficiency, which are rare, and it is important to note that such cases are associated with a bleeding tendency, not thrombosis. Since the Factor V Level assay is not a genetic test, the order failed to trigger a prospective review by the genetic counselling service, another mechanism to check for appropriateness of test orders. Once the Factor $\mathrm{V}$ Level result was available, the provider realized that the wrong test had been ordered. They knew a genetic test was needed and placed an order for the F5 R2 variant. A genetic counselor reviewed this order and modified it to a Factor V Leiden test that reflexed to testing for the F5 R2 variant. Besides the delay in diagnosis, the patient had an uneventful course. The patient was found to be heterozygous for the Leiden variant and R2 variant. Compound heterozygosity of Leiden/R2 adds an additional 10-fold risk of thromboembolic disease. However, an R2 variant by itself adds no additional thromboembolic risk. Hence the F5 R2 variant test is not a first level test for inherited VTE but is only indicated in patients known to have a Leiden variant. Even so, it is often mistakenly ordered at the same time as the Leiden test. Taken together, the exact test and the order in which the testing is performed is important.

There are other instances where similar test names have led to confusion in test ordering. For example, the RET gene has been analyzed in cases of suspected Rett syndrome. The correct gene to the sequence in Rett syndrome is the MECP2 gene, and the RET gene is involved in Multiple Endocrine Neoplasia type 2 (MEN2) disease [10]. Along similar lines, the GLUD1 gene analysis has been sought in cases of suspected GLUT1 deficiency syndrome (caused by mutations in the SLC2A1 gene) (Author personal communication). Thalassemia screen often refers to a test for haemoglobin variants by electrophoresis, but clinicians might expect it to include a haemoglobin gene analysis. Careful naming of tests, along with providing indications for test use in the display name where possible, would help avoid confusion, misuse, and overuse of tests.

\section{Case 2: Direct to consumer (DTC) vs. clinical testing}

A 55-year-old female had an enlarged spleen. During her medical evaluation, she mentioned that her brother had a direct to consumer (DTC) test that identified a heterozygous common variant associated with Gaucher disease, a condition associated with splenomegaly and other features that may include hepatomegaly, anemia, thrombocytopenia and bone anomalies. The provider recommended Gaucher GBA gene testing. The patient requested the same DTC panel her brother had because it was inexpensive and she was interested in other health and wellness information that that test may provide. Blood was collected for the DTC test and sent to the provider's sendouts laboratory for shipment. The laboratory genetic counselor (GC) reviewing test orders contacted the provider to recommend that clinical laboratory testing should be performed rather than DTC testing. Per the institution's Clinical Laboratory Improvement Amendments of 1988 (CLIA) policy, the lab director must approve clinical reference laboratories - the DTC company was not an approved lab. Although this DTC panel did include a few common GBA variants, this panel would miss other GBA common and uncommon pathogenic variants. Full GBA sequencing was the optimal first test based on the patient's ethnic background and sibling's detected variant. There was no clear clinical utility of testing the remaining gene variants included on the DTC test for this patient. The provider confided that he did not want to order the DTC test but wanted to retain his positive relationship with the patient, so ordered it as a first step. He intended to order full GBA sequencing if the DTC panel detected one or no GBA variants. The lab GC discussed that their institution supports patient-centered care, but this is different than patient-driven care: this order felt like the latter. The lab also supports providers in ordering the optimal test for the patient and retaining positive relationships with patients when possible. The provider agreed that GBA gene full sequencing was a more appropriate order for the patient's clinical evaluation. The lab GC empowered the provider to inform the patient that the institution's lab did not approve the DTC order, but she could seek such testing independently and then counsel the patient regarding the utility of GBA full gene sequencing. This transferred the onus of test cancellation to the lab instead of the provider. The GBA sequencing identified one heterozygous common pathogenic variant and one heterozygous uncommon pathogenic variant that would have been missed on the DTC test.

Direct to consumer testing is blurring the lines of clinical testing for providers and patients [11]. While the number of gene variants tested in a DTC test may initially seem like a good monetary value, the cost of the test is unlikely to be reimbursed by insurance payers. Even when DTC testing is performed in labs accredited and certified by the College of American Pathologists and CLIA, the clinical value of the majority of the results can be unclear, especially with very large panels. Such results may necessitate clinical confirmatory testing, thus adding to costs. There is also the risk that a medical provider without genetics expertise may not understand when the test includes targeted variant analysis versus full gene analysis [12]. Taken together, for a patient who has a focused differential diagnosis, it might be best to order genetic testing targeted to that diagnosis from a clinical reference laboratory.

\section{Case 3: Payer reimbursement}

A new-born female presented with congenital heart disease and abnormal facial features, inclusive of cleft lip. The paediatrician did not have much knowledge of genetic syndromes and did not have a genetic counselor or geneticist within 200 miles to consult. The infant had feeding difficulties and required considerable medical support. Upon studying the literature, the paediatrician suspected CHARGE syndrome and ordered $C H D 7$ gene sequencing. The patient's insurance covered this test given it was single gene testing with matching phenotype. The family had a relatively low out of pocket cost. The result of this test was negative.

As the infant grew, she showed motor and language delays. Her parents continued to follow up with the pediatrician every quarter and began early intervention services. Given the infant was on an estimated one-year waitlist for the pediatric geneticist located 200 miles from their home the paediatrician considered a new genetic test that had recently become available and discussed whole-exome sequencing (WES) as a potential genetic test with the family. The pediatrician's medical assistant (MA) called a reputable clinical genetic testing lab who instructed the MA to submit a prior authorization before ordering WES. The MA filled out the correct paperwork and submitted it to the patient's insurance. WES was denied, as the 
payer considered that specific test investigational and experimental. The patient's mother received a call from the paediatrician's office letting her know about this denial and was frustrated by this decision. She spoke with her husband about the possibility of changing insurances at their next enrolment period and began researching other payers. Unfortunately, none of the payers that were available for her or her husband included WES as a covered test.

The child was continuously monitored and at age 4 developed seizures. These quarterly visits to the paediatrician's office caused significant costs to the family's insurance but these visits were justified given the geneticist appointment never occurred as there was still a long waiting list, even 2 years after the referral was made. The patient's mother was then pregnant again, and the parents needed a better understanding of their daughter's diagnosis and outcomes, as well as their chances for recurrence with this pregnancy. Given the family was unable to change their payer and they had still not seen the geneticist, the parents decided to pay out of pocket for WES trio testing: a pathogenic variant in KMT2D was found, diagnosing the child with Kabuki Syndrome. The patient's mother called her payer to inform them that WES was a valuable test that confirmed a rare diagnosis with management guidelines and provided prenatal testing options to their family. The patient's mother encouraged the payer to reconsider coverage for WES for other covered members who may benefit from the same test.

Payer policies play a large role in test selection by both genetics and non-genetic providers. In this case, healthcare dollars were spent on additional testing and provider visits because WES was considered investigational and experimental by the payer and clinical genetics resources were out of reach for the patient. Some payers cover WES because they have completed a systematic assessment of the evidence. This assessment can lead to increased genetic test coverage, reduced spending of healthcare dollars, more appropriate test orders [13], and tailored patient management.

\section{Case 4: Prior authorization}

A 40-year-old male had been experiencing shortness of breath and fatigue. After the presentation to his provider and referral to cardiology, where he underwent an echocardiogram, he was diagnosed with dilated cardiomyopathy. Upon examination of his family history, it was revealed that his mother died at age 35 and a maternal uncle died of heart failure at 50. His cardiologist recommended a dilated cardiomyopathy gene panel as there are many different genes that may explain his personal and family history. Prior authorization is a required process for most genetic tests. In order to obtain prior authorization, a patient's clinical records demonstrating a need for a genetic test as well as evidence that test results would change medical management must be provided to the payer for review and possible approval. The genetic counselor within the hospital laboratory obtained prior authorization for this testing with the required documentation, including the test's Current Procedural Terminology (CPT) billing codes from the outside reference laboratory's website. Despite the prior authorization, payment for the testing was ultimately denied by the patient's health plan. This denial occurred because the billing codes in the claim were not consistent with the billing codes from the testing lab's website that were submitted for prior authorization. The patient was now responsible for payment for a test that was already authorized by the payer.

In this case example, the reference laboratory provided one plausible CPT code that insurance payers are likely to approve on their outwardfacing website but changed to a different plausible CPT code for the direct insurance billing. The laboratory website disclaimer suggests that the billing codes listed on their website may not be the codes that are ultimately included with a claim, but this does not help the patient avoid unreimbursed charges. A review of other reference laboratories' websites revealed that two different laboratories list different billing codes for a similar dilated cardiomyopathy gene panel, e.g., one laboratory submits four billing codes and the other submits one billing code [14,15]. The provider may not understand this variation in the process from lab to lab, and a family may receive unnecessary charges when the performing lab uses CPTs that are different from their insurance payers' approved CPT list. This creates an unfair disadvantage for patients seeking similar medical care.

Special expertise is often required to identify these nuanced reasons for the denial. Collaborative efforts between all stakeholders are needed to develop a transparent CPT billing and prior authorization process that minimizes waste and maximizes value within the healthcare system.

\section{Discussion}

The cases described in this review illustrate multiple factors that impact the genetic testing process and some of the opportunities where laboratory stewardship by individuals with genetic expertise can add value (Figure 1). Figure 1 represents stages in the genetic testing process that require scrutiny for the success of the entire process of laboratory testing. These stages include optimal test selection and ordering for the patient's suspected diagnosis, determining whether appropriate steps have occurred to ensure payer authorization, navigating patient-driven test requests, and weighing testing alternatives when payer authorization is not granted. Even if all needed tasks have been performed to ensure that the best test for the patient is performed within the context of payer authorization, subsequent events can affect whether the patient will be responsible for the bill incurred by the test that was ordered.

Healthcare providers, patients, insurance payers, health systems, and laboratories are all stakeholders in the genetic testing process and outcomes therein. Additional resources are needed to inspire processes where all stakeholders have a greater opportunity to benefit. Genetic counselors can often provide a communication bridge among these stakeholders within a laboratory stewardship program. Collaborative communication between laboratories and payers can facilitate shared strategies to create policies for coverage and CPT coding of medically necessary genetic tests to alleviate billing discrepancies and other barriers to test reimbursement [16].

Billing and reimbursement of genetic testing is a complicated and opaque process. Multiple variables prevent both the ordering provider and the patient from making an informed financial decision and ultimately obtaining optimal insurance coverage for tests. While test coverage approval or denial may at first appear straightforward, variability in CPT billing code interpretation and use creates opportunities for confusion. Analyzing the payer's denial information can guide the provider in determining whether the denial was due to specific medical record details that did not provide medical necessity evidence or whether the billing codes themselves were a barrier to coverage. Follow up of claims data can prompt additional communication between payers and laboratories to confirm coverage outcomes. 
Many insurance payers are heavily burdened with navigating waste and fraud within the healthcare system [17]. Laboratories struggle with antiquated billing systems that often do not provide an accurate representation of the testing performed, making reimbursement of their services complicated and can add to fraud and waste within the system. The large and increasing number of genetic tests are ordered using a computerized order entry system that provides limited information beyond the test name that can lead to confusion such as is demonstrated in the Factor $\mathrm{V}$ Leiden case above. Miscellaneous test orders add further opportunities for errors in ordering. Providers in a busy clinic setting may not have time to access informative resources such as a test catalog or consult with their genetics colleagues to obtain guidance about test selection [18]. Additionally, unique contracts between laboratories, health systems, and payers create variability in billing codes and reimbursement that add complexity to the process. The policies in place to limit fraud and waste can also impair the ability of providers to navigate this system to successfully obtain a testing for their patients and is an opportunity for stakeholders to address how working more transparently together can improve the system as a whole.

As genetic testing is constantly evolving and becoming more applicable to all areas of medicine, the evidence of the utility of a test may not be published at the time of testing. A more individualized evaluation of the medical necessity of that test for a patient may need to occur as genetic testing is increasingly utilized in personalized medicine. The partnership between provider and payer is an important building block in providing tools to navigate optimal testing options for patients. The skills of genetic counselors, including genetic test review and understanding of payer policies, can be utilized to establish working relationships between different stakeholders involved in payer policy development.

The limitations presented by the current billing and reimbursement system itself can present an additional impediment to the timely adaptation to the changing landscape of genetics. Our case study review provides insight into the common pathways in which optimal testing and reimbursement failures occur. Health systems can implement a laboratory stewardship program to shore up those missed opportunities. We propose that stakeholders seek opportunities to collaborate to improve the system.

\section{Acknowledgements}

We thank BassemBejjani, Jessie Conta, and Amanda Elms for input and comments on the article. This work was supported by funds from the Texas Children's Hospital.

\section{References}

1. Phillips KA, Deverka PA, Hooker GW, Douglas MP. 2018. Genetic test availability and spending: where are we now? Where are we going? Health Aff (Millwood) 37(5):710-716.https:// doi.org/10.1377/hlthaff.2017.1427

2. UnitedHealth Center for Health Reform and Modernization. 2012. Personalized medicine: trends and prospects for the new science of genetic testing and molecular diagnostics. 2012. UnitedHealth Group website.

3. Douglas MP, Parker SL, Trosman JR, Slavotinek AM, Phillips KA. 2019. Private payer coverage policies for exome sequencing (ES) in pediatric patients: trends over time and analysis of evidence cited. Genet Med 21(1):152-160.https://doi.org/10.1038/s41436-018-0043-3

4. Cigna. 2020. Whole exome and whole genome sequencing. Medical coverage policy 0519

5. Aetna. Genetic testing. Clinical policy bulletin 0140 .

6. UnitedHealthCare. 2019. Whole exome and whole genome sequencing, UnitedHealthCare Community Plan, medical policy CS150.E

7. Mathias PC, Conta JH, Konnick EQ, Sternen DL, Stasi SM, et al. 2016. Preventing genetic testing order errors with a laboratory utilization management program. Am J Clin Pathol 146(2):221-226.https://doi.org/10.1093/ajcp/aqw105

8. Conway ME, Kalejta CD, Sternen DL, Singh IR. 2020. The Importance of Genetics Experts in Optimizing Genetic Test Orders Through Prospective and Retrospective Reviews. Am J Clin Pathol 153(4):537-547.https://doi.org/10.1093/ajcp/aqz188

9. Dickerson JA, Conta JH, Lockwood CM. 2017. Utilization Management of Genetic Testing. In: Lewandrowski K, (eds). Utilization Management in the Clinical Laboratory and Other Ancillary Services. Springer International Publishing, Switzerland. pp:219-233.

10. Riley J, Stoll K. 2019. Blurred Lines: Comparing Direct-to-Consumer and Clinical Testing. Clinical Laboratory News.

11. Tandy-Connor S, Guiltinan J, Krempely K, LaDuca H, Reineke P, et al. 2018. False-positive results released by direct-to-consumer genetic tests highlight the importance of clinical confirmation testing for appropriate patient care. Genet Med 20(12):1515-1521.https://doi.org/10.1038/gim.2018.38

12. Douglas MP, Parker SL, Trosman JR, Slavotinek AM, Phillips KA. 2019. Private payer coverage policies for exome sequencing (ES) in pediatric patients: trends over time and analysis of evidence cited. Genet Med 21(1):152-60.https://doi.org/10.1038/s41436-018-0043-3

13. Dilated Cardiomyopathy NGS Panel. Fulgent Diagnostics.

14. Dilated Cardiomyopathy Panel. GeneDx

15. Patient-centered Laboratory Utilization Guidance Services (PLUGS). Insurance Alignment.

16. Healthcare Fraud Prevention Partnership. 2018. Examining Clinical Laboratory Services: A Review by the Healthcare Fraud Prevention Partnership. May 2018.

17. 360Dx. 2019. Myriad Genetics Settles Improper Medicare Billing Complaint for \$9.1M. July 19, 2019 